\section{High prevalence of smoking among patients with suspected tuberculosis in South Africa}

\author{
L. Brunet*, M. Pai*, V. Davids", , D. Ling*, G. Paradis*, L. Lenders ${ }^{\#, \mp, ~ R . ~ M e l d a u ", ~}$

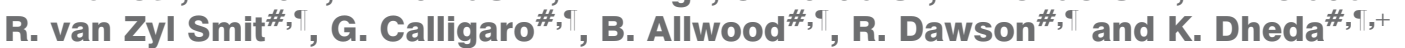

ABSTRACT: There is growing evidence that tobacco smoking is an important risk factor for tuberculosis (TB). There are no data validating the accuracy of self-reported smoking in TB patients and limited data about the prevalence of smoking in TB patients from high-burden settings.

We performed a cross-sectional analysis of 500 patients with suspected TB in Cape Town, South Africa. All underwent comprehensive diagnostic testing. The accuracy of their self-reported smoking status was determined against serum cotinine levels.

Of the 424 patients included in the study, 56 and $60 \%$ of those with active and latent TB infection (LTBI), respectively, were current smokers. Using plasma cotinine as a reference standard, the sensitivity of self-reported smoking was $89 \%$. No statistically significant association could be found between smoking and active TB or LTBI.

In Cape Town, the prevalence of smoking among patients with suspected and confirmed TB was much higher than in the general South African population. Self-reporting is an accurate measure of smoking status. These results suggest the need to actively incorporate tobacco cessation programmes into TB services in South Africa.

KEYWORDS: Cotinine, smoking, South Africa, tobacco, tuberculosis

$\mathbf{T}$ uberculosis (TB) is one of the most important infectious diseases in the world in terms of morbidity and mortality. In 2008, the number of deaths due to TB was estimated at 1.8 million by the World Health Organization (WHO) [1]. In 2008, an estimated 0.48 million new cases occurred in South Africa and, currently, the country ranks third after India and China in terms of total number of annual incident cases of TB [1]. Significantly, $\sim 20 \%$ of patients treated for TB in South Africa have had previous TB [2].

Another important global health concern is tobacco smoking. Smoking is extremely common, with approximately one-third of the population aged $\geqslant 15$ yrs smoking tobacco [3]. It is also one of the most important risk factors for human disease and one of the most frequent causes of death in the world. WHO estimates that tobacco smoking is currently responsible for the death of one in 10 adults worldwide, or of 5 million deaths each year [4]. The tobacco epidemic is evolving alarmingly fast. Although smoking rates have decreased in developed countries over the last part of the 20th century, rates have increased significantly in developing countries [5]. Currently, $82 \%$ of smokers live in low- and middleincome countries [3,6]. In 2003, 35\% of males and $10 \%$ of females smoked daily or occasionally in South Africa [7].

The epidemics of tobacco smoking and $\mathrm{TB}$ are colliding and there is increasing evidence that smoking is an important risk factor for $\mathrm{TB}$ infection, disease and mortality [8-10]. Recently, LÖNNROTH and RAVIGLIONE [11] computed the population attributable risk (PAR) for several risk factors in 22 high TB-burden countries and estimated that active smoking is responsible for $23 \%$ of the TB incidence. Therefore, smoking would account for more TB cases than HIV infection, for which the TB PAR is $19 \%$.

Although there is a significant association between smoking and $\mathrm{TB}$, there are several confounders in data interpretation, including reporting bias, i.e. the reliability of self-reported smoking status. Moreover, data from Africa are limited and how HIV infection interacts with smoking to modulate $\mathrm{TB}$ risk is unclear.

\section{AFFILIATIONS}

*Dept of Epidemiology, Biostatistics and Occupational Health, McGill University, Montreal, QC, Canada, \#Lung Infection and Immunity Unit, Division of Pulmonology and Clinical Immunology,

"UCT Lung Institute, Dept of Medicine, and

${ }^{+}$Institute of Infectious Diseases and Molecular Medicine, University of Cape Town, Cape Town, South Africa.

CORRESPONDENCE

K. Dheda

Dept of Medicine,

H floor (Old Main Building)

Groote Schuur Hospital

Observatory

Cape Town

7925

South Africa

E-mail: keertan.dheda@uct.ac.za

Received:

Aug 262010

Accepted after revision:

Oct 262010

First published online:

Dec 092010

European Respiratory Journal Print ISSN 0903-1936 Online ISSN 1399-3003 
Furthermore, little is known about the association between smoking and recurrent TB. We therefore performed a study to: 1) estimate the prevalence of tobacco smoking in patients with suspected TB in Cape Town, South Africa; 2) measure the sensitivity and specificity of their self-reported smoking status among these patients using plasma cotinine as the reference standard; 3) evaluate the association of smoking with latent TB infection (LTBI), active TB and recurrent TB; and 4) evaluate how HIV modulates these parameters.

\section{METHODS}

\section{Source of data}

Cross-sectional data from a cohort of 500 males and females with suspected TB and prospectively recruited in three clinics in Cape Town, were analysed. The cohort was recruited to study the utility and accuracy of several new TB diagnostic tests in a high TB and HIV incidence setting.

\section{Study population}

To qualify as a patient with suspected TB, individuals aged $\geqslant 18$ yrs had to present with at least one of the following symptoms: persistent cough ( $>2$ weeks), night sweats, coughing up sputum, persistent fever ( $>2$ weeks), unintentional weight loss, being bedridden/unable to walk or loss of appetite.

\section{Measurement of exposure to tobacco smoking}

After informed consent was obtained, all patients completed standardised interviewer-administered questionnaires by one of two trained registered nurses. Four questions pertained to smoking: 1) self-reported current smoking status (Current cigarette smoker? Yes/No); 2) self-reported previous smoking status (Previous cigarette smoker? Yes/No); 3) number of cigarettes smoked per day; and 4) age at which the participant started or quit smoking, if relevant. Current smokers were defined as patients who reported that they were current cigarette smokers. Ever-smokers were defined as patients who reported being either current or previous smokers. Plasma cotinine levels were used to validate the information gathered with the questionnaire. Cotinine measurement was performed by ELISA (Cotinine Direct ELISA Kit CO096D; Calbiotech, Spring Valley, CA, USA) on frozen plasma samples stored at $-80^{\circ} \mathrm{C}$ and thawed immediately before the test. A cut-off of $15 \mathrm{ng} \cdot \mathrm{mL}^{-1}$ was used to determine smoking status, based on the recommendations of the Society for Research on Nicotine and Tobacco [12]. Three technicians, blinded to the self-reported tobacco status and final TB diagnosis, performed the tests.

\section{Measurement of TB outcomes}

All patients underwent extensive diagnostic testing to determine their TB status, which included sputum smear acid-fast bacilli (AFB) microscopy (on expectorated as well as induced sputum), sputum mycobacterial culture and interferon- $\gamma$ release assays (IGRAs) for TB infection (QuantiFERON ${ }_{\circledast}$ TB Gold; Cellestis Ltd, Carnegie, Australia; and T-SPOT®.TB; Oxford Immunotec, Oxford, UK), chest radiograph (evaluated by two independent radiologists) and HIV test (if the patient was not known to be HIV infected). Patients were classified into five possible outcomes of the spectrum of TB infection and disease. 1) "No LTBI and no TB": negative IGRA results, chest radiographs showing no sign of previous or active TB disease, and negative smear and culture results; 2) "LTBI": positive results for at least one IGRA, chest radiographs may or may not show sign of previous TB but no sign of active TB, negative smear and culture results; 3) "Inactive TB": negative IGRA results, chest radiographs showing signs of previous $\mathrm{TB}$, and negative smear and culture results (this differs from LTBI where changes of previous TB are accompanied by a positive IGRA); 4) "Active TB": chest radiographs showing signs of active TB and/or positive smear results and/or positive culture results; and 5) "Unable to classify": if patients could not be classified into one of the other groups due to missing data.

\section{Statistical analysis}

Participants who failed to provide information on their current smoking habit and those for whom plasma samples were not available at the time of cotinine concentration testing were excluded from the analysis. The prevalence of smoking in the study population was estimated with exact $95 \%$ confidence intervals (CIs). The prevalence of self-reported current, past and ever-smoking was estimated for the whole study population, for participants with a history of TB and for each group in the TB spectrum. Three types of smoking measures were used: high levels of plasma cotinine, self-reported current smoking and self-reported ever-smoking (current and previous smokers). Sensitivity and specificity of self-reported current smoking was computed with $95 \%$ CIs with the level of plasma cotinine as the reference standard and using a $15 \mathrm{ng} \cdot \mathrm{mL}^{-1}$ cutoff. The presence of an association between smoking and TB infection or disease was explored using logistic regression models, using either cotinine levels or self-reported eversmoking as the exposure. Data analyses were performed using STATA version 11 (StataCorp., College Station, TX, USA) and OpenEpi version 2.3 (www.OpenEpi.com).

\section{RESULTS}

\section{Study population}

A total of 500 patients with suspected TB were recruited in the principal study. Of these, 76 patients were excluded from the current analysis as a result of the absence of information on their smoking status; thus, the study population consisted of 424 patients with suspected TB.

The sociodemographic and medical characteristics of the study population $(n=424)$ were compared with those of the excluded patients with suspected TB $(n=76)$ and no significant difference between the two groups was found. Patients with and without a history of previous TB were also compared this way. Most characteristics were very similar in both groups, but more patients with no history of previous TB were younger and more likely to be females, abuse alcohol and receive a salary than patients with a history of previous TB. The information on baseline characteristics of the included patients with suspected TB is summarised in table 1 .

The mean age (range) of the study population was 39.5 (18$82)$ yrs. The study population was predominantly male $(67 \%)$ and black African (71\%). Approximately one-third of participants were HIV infected (28\%) and another third (36\%) reported having previously suffered from TB. 
TABLE 1 Sociodemographic characteristics of patients with suspected tuberculosis (TB) included in the analysis and stratified by history of previous TB

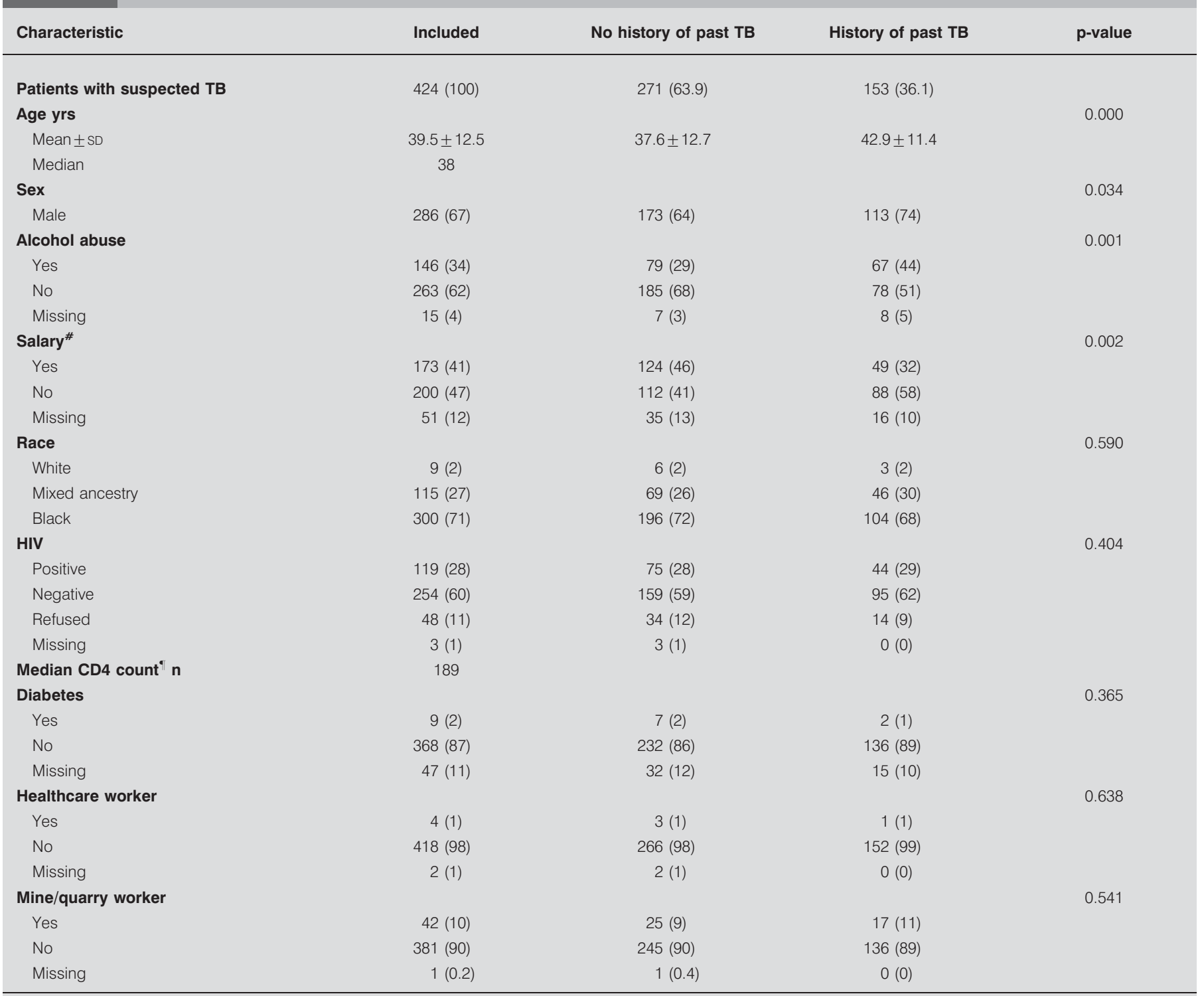

Data are presented as $n(\%)$, unless otherwise stated. ${ }^{*}$ : Subjects were asked whether or not they receive regular salary; ${ }^{\bullet} \mathrm{n}=114$.

Self-reported smoking characteristics among patients with suspected TB

On average, current smokers smoked 10 cigarettes $\cdot$ day $^{-1}$ for 21.5 yrs (median $19 \mathrm{yrs}$, translating into a 11.9 pack-yr smoking history). Ex-smokers smoked, on average, 12 cigarettes $\cdot$ day $^{-1}$ (12.7 pack-yrs) and had quit an average 7.3 yrs prior to enrolment. These characteristics did not vary significantly across the TB outcomes. However, females on average smoked less and started or quit more recently than males.

A total of 153 subjects reported having already suffered from active TB before they were recruited in the study. Because smoking is considered to be a risk factor for $\mathrm{TB}$, smoking habits were evaluated among patients with a history of past TB. The majority $(65 \%)$ were current smokers and $17 \%$ had previously quit smoking. Moreover, significantly more patients with a history of previous TB were current or ever-smokers than patients with no history of past TB $(\mathrm{p}<0.05)$. Among HIVpositive and HIV-negative patients, the prevalence of smoking was similar in those with or without a history of previous TB.

\section{Smoking prevalence across the spectrum of TB infection and disease}

Figure 1 shows the distribution of self-reported smokers in the study population. As outlined in table 2, among patients with suspected TB, $5 \%$ had no TB infection or disease, $20 \%$ had LTBI, $4 \%$ had inactive TB, and $67 \%$ had active TB. Smoking prevalence was generally high across all groups of the spectrum; thus, no pattern could be identified. Using self-report, the prevalence of current smokers was $60 \%$ in those with presumed LTBI and $56 \%$ in active TB patients. 


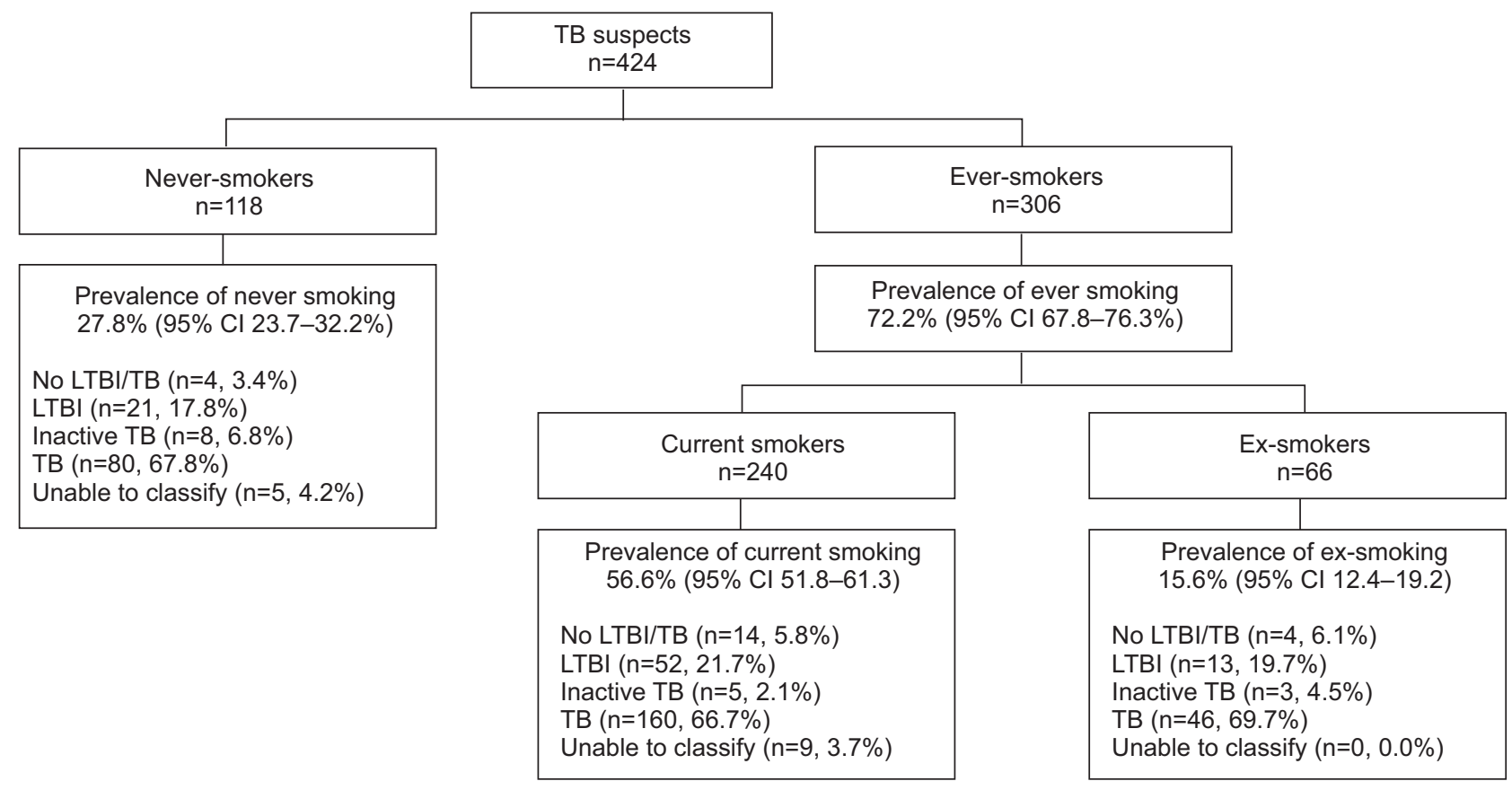

FIGURE 1. Prevalence of smoking among patients with suspected tuberculosis (TB). LTBI: latent TB infection.

Overall, 29\% of previous smokers (19 subjects) reported having quit smoking within 12 months of being recruited in the study. Of these recent quitters, $74 \%$ had active TB and $53 \%$ had smear-positive TB. The prevalence of smoking by TB outcomes is further broken down in table 3 by sex and HIV status. Overall, the proportion of females smoking was lower than that of males, especially in the LTBI and active TB groups.

\section{TABLE 2 Prevalence of smoking across the spectrum of tuberculosis (TB) for different measures of smoking}

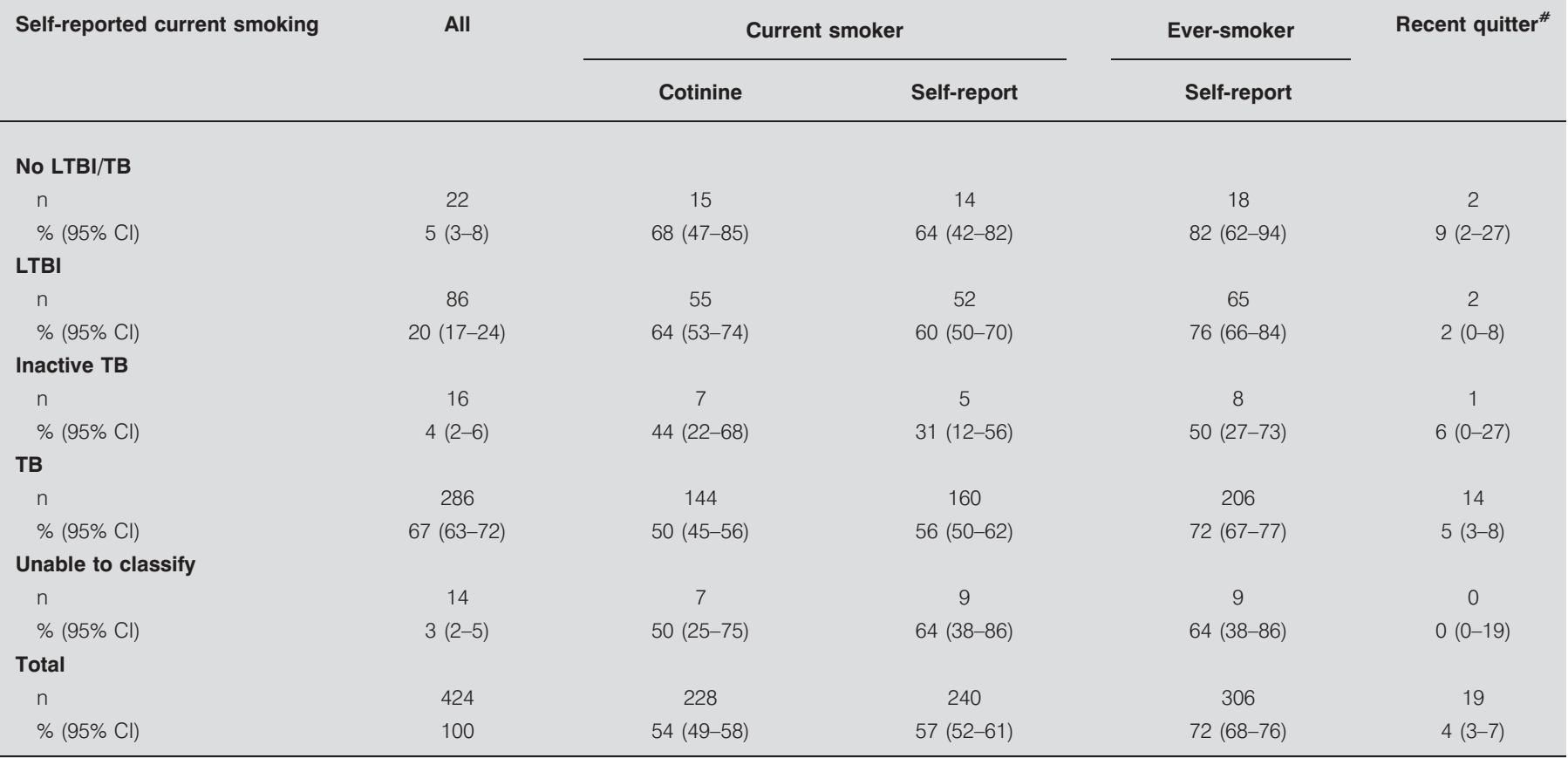

LTBI: latent TB infection. ${ }^{*}$ : ex-smokers who quit in the 12 months preceding admission to the cohort. 
TABLE 3 Prevalence of smoking across the tuberculosis (TB) spectrum stratified by sex and HIV status

\begin{tabular}{|c|c|c|c|c|c|}
\hline Total $\mathbf{n}$ & 424 & 286 & 138 & 254 & 119 \\
\hline Smoker $\mathrm{n} / \mathrm{N}$ & $14 / 22$ & $10 / 15$ & $4 / 7$ & $9 / 13$ & $5 / 7$ \\
\hline \% (95\% Cl) & $68(47-85)$ & $67(41-87)$ & 57 (22-88) & 69 (41-89) & 71 (33-95) \\
\hline \multicolumn{6}{|l|}{ LTBI } \\
\hline \multicolumn{6}{|l|}{ Inactive TB } \\
\hline Smoker $\mathrm{n} / \mathrm{N}$ & $7 / 16$ & $3 / 9$ & $2 / 7$ & $1 / 6$ & $4 / 8$ \\
\hline$\%(95 \% \mathrm{Cl})$ & $44(22-68)$ & 33 (9-67) & $29(5-67)$ & $17(1-59)$ & 50 (18-82) \\
\hline \multicolumn{6}{|l|}{ TB } \\
\hline Smoker n/N & $144 / 286$ & $114 / 189$ & $46 / 97$ & $106 / 175$ & $35 / 78$ \\
\hline \multicolumn{6}{|l|}{ Total } \\
\hline Smoker n/N & 228/424 & 179/286 & $61 / 138$ & $155 / 254$ & $57 / 119$ \\
\hline$\%(95 \% \mathrm{Cl})$ & $54(49-58)$ & $63(57-68)$ & $44(36-53)$ & $61(55-67)$ & $48(39-57)$ \\
\hline
\end{tabular}

LTBI: latent TB infection.

\section{Validation of self-reported smoking status with cotinine levels}

The prevalence of current smoking was estimated at 54\% (95\% CI $49-58 \%$ ) by plasma cotinine and $57 \%$ (95\% CI $52-61 \%$ ) by self-report (difference not statistically significant). The sensitivity and specificity of self-reported smoking was 89\% (95\% CI $84-93 \%)$ and $81 \%$ (95\% CI 75-86\%) respectively, using plasma cotinine as a reference standard.

Because of the high number of recent quitters in the active TB group, the self-reported smoking status of that group was also validated with cotinine levels. In active TB patients only, the sensitivity was $89 \%$ (95\% CI $83-94 \%$ ) and the specificity was $78 \%$ (95\% CI 70-84\%).

\section{Association between smoking and latent, active or recurrent TB}

After adjusting for the potential confounding factors, no statistically significant association could be found between LTBI or active TB and confirmatory cotinine levels, or between LTBI or active TB and self-reported 'ever-smoking' as shown in tables 4 and 5 . Stratification by sex and HIV status did not alter the results (table 5). A stratified analysis of LTBI versus no LTBI could not be performed due to the small number of subjects.

A total of 113 patients with a history of previous TB were diagnosed with active TB and were therefore considered to have recurrent TB. When a logistic regression was performed to evaluate the association between smoking and recurrent $\mathrm{TB}$, no measure of effect was found to be statistically significant.

\section{DISCUSSION}

The key findings of this study were: 1) the very high rates of smoking (56\%) in patients with active TB in Cape Town;
2) self-report is an accurate measure of smoking status among subjects with suspected TB given that only $\sim 10 \%$ of subjects with high plasma cotinine concentration denied smoking; 3) the rates of smoking in those with LTBI, as defined by quantitative T-cell assay readouts, was high (60\%); and 4 ) rates of smoking did not differ by HIV status and was equally high in HIV-positive patients.

The prevalence of self-reported current smoking in the study population was $57 \%$, which is very high in comparison with the 2003 estimate for the South African population [13]. Moreover, the prevalences of smoking of $63 \%$ in males and $44 \%$ in females observed in this study are much higher than the 35 and $10 \%$, respectively, estimated in the general population [7]. This was surprising, since the majority of the study population was black Africans. Black South Africans

\begin{tabular}{|c|c|c|}
\hline $\begin{array}{l}\text { Associati } \\
\text { infection }\end{array}$ & $\begin{array}{l}\text { etween latent } \\
\text { l) and two me }\end{array}$ & $\begin{array}{l}\text { culosis (TB) } \\
\text { s of smoking }\end{array}$ \\
\hline LTBI versus no LTBI/TB\# & $\begin{array}{l}\text { Unadjusted OR } \\
\qquad(95 \% \mathrm{Cl})\end{array}$ & $\begin{array}{l}\text { Adjusted } \\
\text { OR }(95 \% \mathrm{Cl})\end{array}$ \\
\hline \multicolumn{3}{|l|}{ Cotinine levels } \\
\hline$<15 \mathrm{ng} \cdot \mathrm{mL}^{-1}$ & 1.0 (ref.) & 1.0 (ref.) \\
\hline$\geqslant 15 \mathrm{ng} \cdot \mathrm{mL}^{-1}$ & $0.83(0.30-2.25)$ & $0.64(0.14-2.79)$ \\
\hline \multicolumn{3}{|l|}{ Self-reported smoking } \\
\hline Never & 1.0 (ref.) & 1.0 (ref.) \\
\hline Ever & $0.69(0.21-2.26)$ & $0.59(0.08-4.40)$ \\
\hline
\end{tabular}

Ref.: reference value. ${ }^{\#}:$ LTBI $(n=86)$ and no LTBI/TB $(n=22) ; "$ : adjusted for age, sex, alcohol abuse and socioeconomic and HIV status. 
have the lowest rate of smoking in South Africa (23\%) compared with mixed ancestry people (49\%) [14]. More importantly, irrespective of the measure of smoking used, the prevalence of smoking was very high, and remained so across the spectrum of TB diagnoses (active TB, LTBI and when these categories were stratified by HIV status). These data suggest that the South African National TB Programme (NTP) and the NTPs of other African governments, given the associated increased mortality in smokers with TB, should introduce policies and interventional measures to curb smoking among TB and TB-HIV co-infected patients. These measures should be integrated with TB-HIV control programmes together with improved case-finding strategies, infection control, isoniazid chemoprophylaxis, DOTS, and improved access to antituberculous drugs and improved diagnostics. Simple smoking cessation interventions are possible in resource-poor settings in Africa [15]. In Indonesia [16] and Kerala (India) [17], the majority of $\mathrm{TB}$ patients quit smoking during their $\mathrm{TB}$ treatment, although relapse rates were high. Controlled trials are now required to assess the impact of such interventions on smoking rates and TB outcomes.

It has been argued that published studies highlighting the association between smoking and TB are confounded by several limitations, including reporting bias [18]. However, our findings are reassuring in that self-reporting is an accurate method of data collection when measured against plasma cotinine as the reference standard, at least in a South African setting. The error margin of self-reporting as a measure of smoking is also useful when planning and analysing existing and future TB-smoking-related studies in Africa.

Only $11 \%$ of smokers failed to disclose their status when cotinine levels were elevated, presumably due to shyness or discomfort. However, it is possible that this number may be even smaller, as the cotinine reference standard may have overestimated the number of true smokers. This may have been due to detection of passive smoking (second-hand smoke) in nonsmokers [18]. Participants in this study come from resource-poor backgrounds where there is overcrowding and poor ventilation in small informal dwellings and in congregate community settings. Thus, the effect of passive smoking may be greatly enhanced accounting for appreciable detection of cotinine in nonsmokers. Although less common, another possible source of overestimation defined by the reference standard could be tobacco-chewing or nicotine replacement therapy, since the test detects cotinine in the plasma regardless of its source [18, 19]. By contrast, 19\% of subjects reported being smokers when plasma cotinine was in the normal range. This could have been due to information bias related to stigmatisation of TB. Thus, participants could have falsely reported their status so as to attribute their symptoms to smoking rather than TB. An alternative explanation is related to the half-life of cotinine in blood, which is about 18-20 h and, thus, smokers who did not smoke for $>48 \mathrm{~h}$ may have been missed and erroneously classified as nonsmokers [20-22]. This hypothesis is in agreement with the results obtained by CARABALlo et al. [23], who postulated that observed discrepancies in self-report and cotinine levels could be due to occasional smokers. A further alternative explanation is protopathic bias or 'sick quitter bias', wherein a worsening of health status may cause a change in behaviour [24]. It is well 
known that when smokers start experiencing respiratory symptoms they tend to quit [25]. Hence, subjects with suspected TB may have quit smoking shortly before attending the clinic but still reported they were current smokers. Indeed, 14 out of the 19 patients with suspected TB quit smoking $<1 \mathrm{yr}$ before being recruited.

A very high proportion of active TB cases were diagnosed in this cohort $(67 \%)$. Participants were recruited because they presented the signs and symptoms of active TB, explaining the high number of active cases.

In contrast to several studies, we failed to find an association between smoking and active TB [8-10, 26-33]. In our study, the association between cotinine levels/self-reported 'ever-smoking' and TB could have been obscured by the differential effect of several co-factors, including malnutrition, alcohol abuse, poverty, overcrowding, strain type and transmission dynamics, that led to very high rates of $\mathrm{TB}$ in nonsmokers. Furthermore, 'sick quitter bias' could have contributed to this effect by under-estimating the true smoking rates in TB patients when cotinine levels were used to measure smoking. However, this should not have confounded the association between "ever smoking" and TB. Thus, the absence of a statistically significant association between smoking and TB cannot be interpreted as evidence against the hypothesis of a relationship. For similar reasons, in contrast with other studies [8-10, 26-33], we found no relationship between smoking and LTBI. This is the first study to comprehensively evaluate the relationship between standardised quantitative T-cell responses and smoking. The effect of defining LTBI by T-cell assay responses, rather than by the tuberculin skin test, on the association remains unclear.

Our study has several limitations, including potential misclassification of exposure due to self-report. The use of cotinine concentration has its own limitations, including the detection of tobacco chewers and patients using NRT, and failure to detect those who had not smoked for $>48 \mathrm{~h}$. The effect of used freeze-thawed samples is unclear but is thought to be minimal [34]. The questionnaire used contained no information on other forms of tobacco intake and limited the self-reported smoking information available in the database. In addition, having only four questions on smoking may have failed to capture the behaviour in a comprehensive way. However, for practical reasons, we preferred to keep the time taken for capture of information as short as possible so as not to compromise our capacity to recruit patients for the diagnostic component of the study. Since smoking can induce coughing, the prevalence of smoking found in this study might have been influenced by the selection of participants, given that the presence of a persistent cough was part of the inclusion criteria. Finally, the results we report may only be generalised to South Africa. Nevertheless, they provide valuable information for the development of policies in South Africa and other high HIV prevalence settings.

In summary, our findings indicate high rates of smoking in patients with LTBI and active TB and that self-report is a reliable tool to estimate the incidence of smoking within a community. NTPs in South Africa and other high-burden settings should include investigation of smoking behaviour and offer smoking cessation interventions in the integrated TB-HIV clinic setting. Such programmes could also be beneficial in preventing many other smoking-related conditions. Such an approach would not only result in important health benefits, but also in considerable financial gains [6].

\section{SUPPORT STATEMENT}

This study was supported by a TBsusgent grant from the European Commission (EU-FP7). RVZS is supported by a Fogarty International Clinical Research Scholars/Fellows Support Centre NIH grant (R24TW007988). This study was supported by a TBsusgent grant from the European Commission (EU-FP7) and the EDCTP. M. Pai is supported by a CIHR New Investigator Award from the Canadian Institutes of Health Research. R. van Zyl Smit is supported by a Fogarty International Clinical Research Scholars/Fellows Support Centre NIH grant (R24TW007988).

\section{STATEMENT OF INTEREST}

None declared.

\section{ACKNOWLEDGEMENTS}

The authors would like to thank A. Benedetti and R. Menzies (Dept of Epidemiology, Biostatistics and Occupational Health, McGill University, Montreal, QC, Canada) for their help.

\section{REFERENCES}

1 World Health Organization. Global Tuberculosis Control - A short update to the 2009 report. Geneva, WHO, 2009.

2 Verver S, Warren RM, Beyers $\mathrm{N}$, et al. Rate of reinfection tuberculosis after successful treatment is higher than rate of new tuberculosis. Am J Respir Crit Care Med 2005; 171: 1430-1435.

3 Jha P, Chaloupka FJ, Corrao M, et al. Reducing the burden of smoking world-wide: effectiveness of interventions and their coverage. Drug Alcohol Rev 2006; 25: 597-609.

4 World Health Organization. Why is tobacco a public health priority? 2009. www.who.int/tobacco/health_priority/en/index. html Date last accessed: October 27, 2009; Date last updated: 2009.

5 World Health Organization. The World Health Report 2002: Reducing Risks, Promoting Healthy Life. Geneva, WHO, 2002; pp. 1-248.

6 Siddiqi K, Lee AC, Siddiqi K, et al. An integrated approach to treat tobacco addiction in countries with high tuberculosis incidence. Trop Med Int Health 2009; 14: 420-428.

7 Peer N, Bradshaw D, Laubscher R, et al. Trends in adult tobacco use from two South African demographic and health surveys conducted in 1998 and 2003. S Afr Med J 2009; 99: 744-749.

8 Lin HH, Ezzati M, Murray M. Tobacco smoke, indoor air pollution and tuberculosis: a systematic review and meta-analysis. PLoS Med 2007; 4: e20.

9 Bates MN, Khalakdina A, Pai M, et al. Risk of tuberculosis from exposure to tobacco smoke: a systematic review and metaanalysis. Arch Intern Med 2007; 167: 335-342.

10 Slama K, Chiang CY, Enarson DA, et al. Tobacco and tuberculosis: a qualitative systematic review and meta-analysis. Int $J$ Tuberc Lung Dis 2007; 11: 1049-1061.

11 Lönnroth K, Raviglione M. Global epidemiology of tuberculosis: prospects for control. Semin Respir Crit Care Med 2008; 29: 481-491.

12 SRNT Subcommittee on Biochemical Verification. Biochemical verification of tobacco use and cessation. Nicotine Tob Res 2002; 4: 149-159.

13 van Walbeek C. Recent trends in smoking prevalence in South Africa - some evidence from AMPS data. S Afr Med J 2002; 92: $468-472$. 
14 Statistics South Africa. Mid-year population estimates. 2009. www.statssa.gov.za Date last accessed: November 9, 2009; Date last updated: July 27, 2009.

15 El Sony A, Slama K, Salieh M, et al. Feasibility of brief tobacco cessation advice for tuberculosis patients: a study from Sudan. Int J Tuberc Lung Dis 2007; 11: 150-155.

$16 \mathrm{Ng} \mathrm{N}$, Padmawati RS, Prabandari YS, et al. Smoking behavior among former tuberculosis patients in Indonesia: intervention is needed. Int J Tuberc Lung Dis 2008; 12: 567-572.

17 Pradeepkumar AS, Thankappan KR, Nichter M. Smoking among tuberculosis patients in Kerala, India: proactive cessation efforts are urgently needed. Int J Tuberc Lung Dis 2008; 12: 1139-1145.

18 World Health Organization. A WHO/The Union Monograph on TB and Tobacco Control: Joining efforts to control two related global epidemics. Geneva, WHO 2007.

19 Gorber SC, Schofield-Hurwitz S, Hardt J, et al. The accuracy of selfreported smoking: a systematic review of the relationship between self-reported and cotinine-assessed smoking status. Nicotine Tob Res 2009; 11: 12-24.

20 Haufroid V, Lison D. Urinary cotinine as a tobacco-smoke exposure index: a minireview. Int Arch Occup Environ Health 1998; 71: 162-168.

21 Binnie V, McHugh S, Macpherson L, et al. The validation of selfreported smoking status by analysing cotinine levels in stimulated and unstimulated saliva, serum and urine. Oral Dis 2004; 10: 287-293.

22 Dhar P. Measuring tobacco smoke exposure: quantifying nicotine/ cotinine concentration in biological samples by colorimetry, chromatography and immunoassay methods. J Pharm Biomed Anal 2004; 35: 155-168.

23 Caraballo RS, Giovino GA, Pechacek TF, et al. Factors associated with discrepancies between self-reports on cigarette smoking and measured serum cotinine levels among persons aged 17 years or older: Third National Health and Nutrition Examination Survey, 1988-1994. Am J Epidemiol 2001; 153: 807-814.

24 Delgado-Rodriguez M, Llorca J. Bias. J Epidemiol Community Health 2004; 58: 635-641.

25 Langhammer A, Johnsen R, Holmen J, et al. Cigarette smoking gives more respiratory symptoms among women than among men. The Nord-Trondelag Health Study (HUNT). J Epidemiol Community Health 2000; 54: 917-922.

26 Pai M, Mohan A, Dheda K, et al. Lethal interaction: the colliding epidemics of tobacco and tuberculosis. Expert Rev Anti Infect Ther 2007; 5: 385-391.

27 Dheda K, Johnson MA, Zumla A, et al. Smoking is not beneficial for tuberculosis. Am J Respir Crit Care Med 2004; 170: 821.

28 van Zyl Smit RN, Pai M, Yew WW, et al. Global lung health: the colliding epidemics of tuberculosis, tobacco smoking, HIV and COPD. Eur Respir J 2010; 35: 27-33.

29 Maurya V, Vijayan VK, Shah A. Smoking and tuberculosis: an association overlooked. Int J Tuberc Lung Dis 2002; 6: 942-951.

30 Davies PD, Yew WW, Ganguly D, et al. Smoking and tuberculosis: the epidemiological association and immunopathogenesis. Trans $R$ Soc Trop Med Hyg 2006; 100: 291-298.

31 Hassmiller KM. The association between smoking and tuberculosis. Salud Publica Mex 2006; 48: Suppl. 1, S201-S216.

32 Chiang CY, Slama K, Enarson DA. Associations between tobacco and tuberculosis. Int J Tuberc Lung Dis 2007; 11: 258-262.

33 Zellweger JP. Tobacco and tuberculosis. Monaldi Arch Chest Dis 2008; 69: 83-85.

34 Riboli E, Haley NJ, De Waard F, et al. Validity of urinary biomarkers of exposure to tobacco smoke following prolonged storage. Int J Epidemiol 1995; 24: 354-358. 